\title{
Adaptation Strategies: A Comparison between E-Learning and E-Commerce Techniques
}

\author{
Bill Vassiliadis ${ }^{1,2}$ and Antonia Stefani ${ }^{1}$ \\ ${ }^{1}$ Educational Content, Methodology and Technology Laboratory (e-CoMeT Lab) \\ Hellenic Open University, Greece \\ ${ }^{2}$ Dept. of Administration of Cooperative Organisations, \\ TEI of Messolonghi, Greece \\ $\{$ bb, stefani\} @eap.gr
}

\begin{abstract}
The importance of e-learning and e-commerce applications has significantly increased in the past few years. Seeking better design and implementation principles is a research goal with, potentially, a significant impact. One of the commonalities of both applications is user-centricity. Understanding user behavior is critical especially in user-centered applications such as e-commerce and e-learning. In this work we discuss some of the fundamental similarities and differences in e-commerce and formal e-learning adaptation and discuss lessons that could be learned. We argue that current user pattern mining techniques should take into account behavioral and educational theories for distance learning in order to be efficient.
\end{abstract}

Keywords: Adaptation strategies, comparison study, E-learning, E-commerce.

\section{Introduction}

The convergence of telecommunication and information technologies has enabled the realization of advanced tools for e-learning, on-line collaboration and content delivery. Two of the most popular applications of web based systems nowadays are elearning and e-commerce. Despite their differences, both types of applications are facing similar challenges: they rely on a "pull" model of information flow, they are hypermedia based, they use similar techniques for adaptation and they benefit from semantic technologies and Knowledge Management. The underlying business models also share the same basic principle: users access digital resources from a distance without the physical presence of a teacher or a seller. Since the importance of both applications has increased, better ways of designing and implementation are needed. Designers, developers and researchers of e-learning systems may learn some useful lessons from the e-commerce domain and vice-versa. There is a lot of common ground between the two types of applications; in this paper the focus is on understanding user behavior and the mechanisms that analyze, model and take advantage of it. 
It is widely acknowledged that understanding user behavior is of paramount importance in such user-centered applications. The similarities of e-commerce and elearning suggest that, at least, some user behavioral patterns are similar to both applications. For this reason, major scientific investments have been made towards understanding how users browse, search, buy, learn and make mistakes on line. This understanding influences both the design and the content of these applications.

E-commerce has a long history of practical use of user modeling mechanisms, however data and techniques used are a corporate secret. They are regularly incorporated in pre- and post sales procedures. On the other hand, it is worth noting that, despite the large number of related publications, limited claims of success of voluntary use of e-learning systems has been reported. The software has the capabilities but user acceptance remains low. Only within the past few years, researchers, originating mainly from the educational domain point out that little has been done for context-aware adaptation. In the case of e-learning, context-aware parameters that seem to have been ignored to date, stem from behavioral and pedagogical theories. From our point of view, a simple but yet important parameter still eludes many e-learning adaptive hypermedia efforts: systems and techniques should be designed by both engineers and educationalists in order to increase user acceptance. In e-commerce, a better job has been done in applying market and psychological theories for attracting and supporting users. The increased revenues of e-commerce is a proof that, at least, it works adequately. There is no strong evidence of how much the understanding of user behavior constitutes to this success (usually the business concept and the quality of goods and services take much of the credit). Building applications based on educational theories seems to be harder because the goals, the users and the support they need are different. Perhaps a kind of "educational marketing" theory should be used to attract learners without compromising the true educational goals.

For the rest of the paper we consider adaptation as the set of techniques that take record, analyze, model and take advantage of user behavior in order to improve user experience. We extend our initial remarks made in [1] to discuss some of the major similarities and differences between e-learning and e-commerce systems (and particularly B2C systems) that affect adaptation and system usability.

\section{Adaptive Hypermedia Systems: State of the Art}

Adaptation in hypermedia systems has followed initially two distinct approaches that later converged: adaptation based on rules and algorithmic methods $[2,6,8]$.

\subsection{Rule Based Adaptation}

Rule based adaptation was dominating the field before algorithmic methods became a trend. A few years ago, most existing systems realized adaptation through the use of pre-determined rules, which assigned adaptation constituents to interaction situations in a rather "arbitrary" way. Decision-theoretic frameworks for run-time adaptation, 
are mostly utility-based decision making techniques in the context of the standard reference model for intelligent hypermedia presentation systems. Other trends examine the efficiency of hybrid rule-based/ algorithmic methods for adaptation.

\subsection{Algorithmic Adaptation}

A very popular, algorithmic approach for discovering user behavior in hypermedia systems is a special area of data mining: web mining. In turn, Web mining is generally focused on content, structure and usage mining. Observe that there is almost a one-to-one correspondence with the adaptation possibilities of hypermedia systems described in section 4 . Web mining relies on standard data mining algorithmic approaches which face two major problems in this particular application domain: the weak relations between users and the hypermedia system, and complicated behaviors. The first problem is created by the fact that users often do not have clear goals or the system does not have enough information about them to adapt to their preferences (as in the case of first time or infrequent users). The second one is caused by the diversity of the user population.

Content mining analyses the content of hypermedia documents using information retrieval techniques. The most popular method for representing and adapting information presentation is the Vector Representation model (e.g. the TFIDF technique), inversion and clustering to categorize / rank documents according to their content. The main idea relies on the existence of both content and context information in the hypermedia environment. Search and retrieval processes are viewed as a combination of methods that exploit both types of information while intermediate results can be cross-referenced using merging algorithms.

Structure mining of hypermedia was largely initiated by significant works in link analysis ranking in the web such as PageRank (used in the Google search engine), HITS and their variations.

Usage mining presented significant results. A special area of web usage mining is collaborative (social) filtering which refers to the categorization users to groups according to their preferences. The most popular methods are Markov models, association rules, sequential patterns, most forward access patterns, tree structure access patterns, clustering and hybrid models [5].

\subsection{Impact of Techniques on Adaptation}

Analysis of user -system interaction has been identified as a major contributor to system usability and adaptability. This contribution was early credited in hypermedia systems which have been accompanied with logging mechanisms. The literature provides a wealth of information about how a log can be pre-processed, analyzed and how user preferences may be grouped and used for all kinds of adaptation tasks. Prediction of user behavior is also valuable for marketing purposes and business intelligence of e-commerce. Research in this field has been expanded to include community support services of hypermedia systems such as newsgroups and forums using special methods based on social metrics. Static user profiles are also a premium 
source of knowledge in most recent implementations of hypermedia applications. Profiling is based mostly on explicit graded user feedback and in some occasions on server logs analysis. Table 1 summarizes the impact of the main approaches.

Table 1. Main adaptation techniques' impact on a hypermedia system

\begin{tabular}{|c|c|c|c|}
\hline METHOD $\boldsymbol{V}$ । Adaptation level $\boldsymbol{|}$ & Content & Layout & Navigation \\
\hline Rule based & & $\nabla$ & $\square$ \\
\hline Algorithmic based & & & $\square$ \\
\hline Content mining & $\square$ & & $\square$ \\
\hline Structure mining & & & $\square$ \\
\hline Usage mining & $\square$ & $\square$ & $\square$ \\
\hline User profile & & $\square$ & $\square$ \\
\hline
\end{tabular}

\section{$3 \quad$ User Modeling: Learner vs. Buyer Models}

\subsection{User Modeling Technology}

User modeling is a popular adaptation method already counting several years of application to a variety of systems [3,7,9]. A user model maintains an explicit and dynamic representation of the user. It represents the system's understanding of a user, and it consists both of known facts (such as personal information) and inferred beliefs based on previous interactions. The user model should be structured and populated, ideally, according to the adaptation requirements of the application (table 2).

Table 2. Adaptation requirements for different hypermedia system configurations

\begin{tabular}{|l||c|c|c|}
\hline Parameter $\boldsymbol{\nabla}$ I Strategy & Aggressive & Neutral & Cooperative \\
\hline Goal & Commercial & Informative & Learning \\
\hline Information load & High & Medium & High \\
\hline Focus & Transactions & Documents & Interaction \\
\hline Information use & Access/evaluate & Access & Access/create/edit/ \\
& & & share \\
\hline Adaptation requirements & High & Medium & High \\
\hline
\end{tabular}

\subsection{Student Models}

E-learning was among the first category of applications, and probably the first among the on-line ones, that used user models for adaptation. Early approaches used machine learning techniques initially developed for the information system domain to support adaptation [4]. The advent of the internet and the spectacular increase of the user population, both in number and diversity, posed the need for new approaches. New 
methods focus on the way in which learners acquire, store, process and share knowledge rather than forcing them to follow a stereotype, predetermined behavior.

Learner (student) modeling has been extensively researched because there is already a strong background on pedagogical theories. The most interesting approaches include the stereotype, the overlay, the perturbation, the analytic, the synthetic and the mixed model. Table 3 summarizes these approaches.

Table 3. Main user modelling methods for e-learning

\begin{tabular}{|l|l|l|}
\hline \multicolumn{2}{|l|}{ Method } & Moderlying Method \\
\hline Overlay & Knowledge construction & Expert based, concept-oriented \\
\hline Perturbation & Knowledge extension & Expert based, perturbation \\
\hline Analytic & Behavioral & Learner based \\
\hline Synthetic & Behavioral & Learner based \\
\hline Stereotype & Fixed rules, static & Learner based, categorization \\
\hline
\end{tabular}

\subsection{Buyer Models}

Static user profiles were the primary source of knowledge in early implementations of adaptive B2C applications. Besides traditional approaches that produce user models based on log file analysis or questionnaires, works worth mentioning are those applied to live help systems for e-commerce web sites and recommender mechanisms.

\section{Adaptation Requirements: E-Learning vs. E-Commerce}

A fundamental question in this comparison study is 'what should be adaptable and how?'. In this section, we explore requirements that affect the design of adaptation mechanisms.

The answer to the question of what can be adapted is mainly of a technological nature, while the one referring to how, a pedagogical - technological one. Content, layout and navigation are the three types of adaptation common in most adaptive hypermedia systems. Adaptation strategies should also be context specific. In the case of e-commerce, they rely on behavioral, social, marketing and other theories. In the case of e-learning, they rely to pedagogical and learning theories.

User behavior differs significantly. B2C user behavior falls under four general categories: directed buying, search/deliberation, hedonic browsing and knowledgebuilding. The first two are goal-oriented, while the other two resemble explorative search behavior. On-line learning behavior, on the other hand, may follow the social/collaborative, contextualized or experiential model. There is a difference in 
complexity but also an interesting point: knowledge building is also used in ecommerce: navigation and hyperlinked data aid collaborative knowledge building which is becoming available for all kinds of adaptive hypermedia systems.

It is obvious that, although both e-learning and e-commerce are largely hypermedia based and use the same internet protocols and technologies to work, user goals differ significantly. Starting from e-learning applications, user goals are, ideally, to reach a set of predefined educational objectives, to learn. These objectives are the same for both the designers and the users of such system. E-learning is closely connected to educational, pedagogical and behavioral theories. Most current applications follow the information transfer paradigm where information is passed from the system to the user. Advanced learning models, the future trends in e-learning, anticipate knowledge construction and sharing and most importantly collaboration through the formation and management of virtual learning communities. Most formal platforms support the on-line presence of tutors (either real persons or avatars). Since e-learning uses online resources in its core mechanism, requirements such as efficient browsing and searching mechanisms are necessary. But how can context be described without the proper metadata? Semantic enriched, context-aware hypermedia may be the solution.

In the case of e-commerce and especially B2C (Business to Consumer) applications, goals are somewhat different for designers and users. Designers follow marketing strategies in order to sell as many products as possible. This includes making the user experience as seamless as possible, recommender systems and automatic offers. User goals are the same as every buyer goals: locate the appropriate product as simply as possible and access as much and relevant information as possible. It is obvious that designers and users, in this context, do not share the same goals in so far as an underlying theory is concerned. Marketing theories are followed by designers for increasing profits, not by users to directly gain benefit. In B2C, information is transferred from the application to the user and there is no direct possibility of knowledge building as in the case of e-learning. There is, however, the support of knowledge sharing through the use of off-line collaboration (mainly forums where buyers exchange opinions). Another difference is the absence of on-line guidance in the strict and formal form of tutoring. Off-line consultation with experts, or on-line support is significant but not as crucial as in the case of e-learning. On-line communities are present in the form of communities of practice and share significant information. They are however, in most cases, informal and communicate mostly by off-line tools.

Both applications are quite demanding in their own way since they serve a diverse user population. Competition in e-commerce is quite fierce and customers are demanding by default. Many surveys have shown that a B2C system should be able to offer to its users the information they want in a relatively small amount of time. Failure to cope with user requests fast often results in increased user dropout rates. Most frequently, users do not return to B2C systems that have not managed to satisfy their requests quickly and reliably. In the case of formal e-learning systems, however, users are obliged to use a given system by formal procedures, no matter how frustrated they are by its inflexibility. It is characteristic that the duration of an ecommerce session is smaller than that of an e-learning session, in fact they term 
"session" is not quite applicable in e-learning. Consequently, flexibility and usability are important requirements of e-learning systems as much as they are for e-commerce, but to serve a different purpose: to assist in reaching the educational objectives and not to maintain the customer base.

New trends in pedagogy concentrate more on constructivism, the building of knowledge by way of social interaction and collaboration on-line. Although constructivism has been identified by many researchers as one of the most appropriate methods for learning, science has not yet comprehended and analyzed the mental processes of human knowledge building, collaboration, sharing, evolution and reuse. Thus, a learner does not behave exactly as a buyer does. Another important parameter is the identification of how users perceive and process information and how they complete tasks. It seems that the "one-size fits all" approach has proved to be relatively successful in e-commerce, which is not the case in e-learning. The number of different learning styles is large, and each of them is largely affected by numerous context parameters known only to the teaching staff. So, traditional adaptation mechanisms are somewhat superficial. One interesting proposal by (Stash et al., 2004) is to let the teaching staff configure adaptation by choosing the appropriate learning style that best describes the educational context. This approach has merit in theory, but it is difficult to be accomplished in practice since such software may become too difficult to use. Flexibility is the key to the success of this approach.

A popular misconception is that adaptation in both e-learning and e-commerce is governed by the same principles. Techniques such as log analysis with the use of machine learning techniques, or general purpose user profiling will do the job in both cases. Although the above mentioned strategies are quire successful in the ecommerce paradigm, this is not always the case in e-learning. Most efforts fail to take under consideration pedagogical models and educational goals. E-learning adaptation in formal systems is more about sequencing learning material and workflow of learning processes. E-commerce recommendation/adaptation mechanisms simply will not do because they rely on common beliefs (preferences) which are often misconceptions and possibly not quite useful pedagogically. For example, the logical structure of a course is not taken into account when links or documents are recommended by an adaptation mechanism. This way, a pedagogically false sequence may be initiated by common user mistakes. E-learning is a procedure that is guided by formal theories, with well defined goals and methods in order to impose some kind of pedagogical control over the learning process.

Parameterization of mining algorithms requires technical experience and as such is a tedious process for educators. For example, many data mining algorithms use thresholds for clustering user sessions. How are these thresholds connected to the educational process, the learning pace of students, the goals and more importantly to subjective pedagogical factors such as knowledge construction and social learning? Furthermore, the new trend of constructivism theory for learning does not explain adequately how people learn: we cannot describe exactly and more importantly model mathematically the exact processes of learning. It is thus extremely difficult at this time to use machine learning techniques that are adequate for efficient adaptation in 
very diverse populations. This is not the case in e-commerce where buyers follow more well-defined processes in order to perform transactions.

It is worth noting that users of hypermedia systems continue using the services as long as the use cost $U$ does not exceed the perceived benefits $B$. Use costs and benefits are subjective factors and cannot be calculated with accuracy. When costs exceed benefits $(U>B)$, then a usability bottleneck appears. It is our opinion that usability bottlenecks are more common in e-learning systems due to their complexity.

Research efforts on adaptation have already bear fruits: real life e-commerce systems, such as the recommender engine of Amazon.com, are successfully providing adaptation in the form of recommendations. Marketing systems are using machine learning techniques to analyze email text and discover user preferences. Business intelligence, an information systems concept, is expanded to include web based systems. A plethora of $\log$ analysis techniques has also appeared in the literature showing good adaptation results. One might expect that e-learning could use directly the same (or slightly modified) methods for adaptation. In the case of adaptation using log analysis this has not happened yet in a large scale. Web based e-commerce and web based e-learning may seem analogous but also have very significant differences. For example, a session in e-learning is very different from a transaction (session) in ecommerce. In the latter case, the session begins with an access to a web page and ends with an order or a logout from the electronic basket. In this case, the session duration is short. An e-learning session may span many access sessions (e.g. access forums, download exercises, take online tests etc.). Collaboration, and thus links between users, is stronger in e-learning. For example forums are more active in e-learning than in an e-shop.

Besides the obvious advances in user behavior mining, the missing link with the underlying theories (either pedagogical or behavioral) has already been pin-pointed by researchers: crude data mining alone is not enough since this kind of algorithmic techniques group objects according to 'frequency and/or confidence constraints'. A solution that has been proposed is the use of data mining languages that guide the basic mining algorithms to mine user behavior patterns. It is our opinion that this approach is complicated and this is the reason why it has not been used widely by the e-learning community.

Hypermedia also include new interfaces used for real time collaboration: on-line chat, video conferencing, virtual class tools, Virtual Scientific Experiments etc. Little research has been done on the adaptation of such tools. Another domain for research is service oriented computing and more particularly, web services. Web services with a hypermedia interface may be recorded as a new generation of hypermedia systems. Application software is (or will) gradually transform to 'software as a service'. In such a case the functionality of a system is performed by a set of cooperating services, each service being a building block to a large service oriented construction. The question of "what and how to adapt" posed before, is partially restated for upcoming hypermedia service - oriented architectures. Since, however standards for web services are not yet fully delivered, it would not be safe to draw any conclusions at this time. There are many other differences that drive the diversity of user behavior in e-learning and ecommerce. Table 4 summarizes the most important ones in our opinion. 
Table 4. A summary of factors that affect user behaviour diversity for e-commerce and e-learning

\begin{tabular}{|c|c|c|}
\hline Parameter & E-Commerce & E-Learning \\
\hline Goal & $\begin{array}{c}\text { Complete financial } \\
\text { transaction: getting a product }\end{array}$ & Reach learning objectives \\
\hline Session duration & One session & Multiple sessions \\
\hline GUI & $\begin{array}{l}\text { Text, multimedia, graphics - } \\
\text { animation, video, sound }\end{array}$ & $\begin{array}{l}\text { Audio-conferencing, webcast, } \\
\text { multimedia, chat, simulation, white- } \\
\text { boarding, text, video - conferencing, } \\
\text { animation }\end{array}$ \\
\hline Adaptation & Presentation & Sequence \\
\hline Feedback & $\begin{array}{l}\text { From the system (help, } \\
\text { FAQ), administrator (e-mail, } \\
\text { telephone) }\end{array}$ & $\begin{array}{l}\text { From the system (help, FAQ), the } \\
\text { instructor (chat, phone, e-mail, } \\
\text { synchronous collaboration), e-learners } \\
\text { (chat, asynchronous - synchronous } \\
\text { collaboration), portals }\end{array}$ \\
\hline Mining method & Cookies, log analysis & Log analysis, instructors reports \\
\hline Technology & Web based & Web based/ Multimedia \\
\hline Complexity & $\begin{array}{l}\text { E-learner - system - } \\
\text { transaction } \\
\end{array}$ & $\begin{array}{l}\text { E-learner - different collaboration } \\
\text { platforms - learning activity }\end{array}$ \\
\hline Underlying method & Marketing & Pedagogical \\
\hline Network & Client-Server & Client-Server, $\mathrm{P} 2 \mathrm{P}$ \\
\hline Drop out factors & Information stress & Competency, grading \\
\hline Main tools & $\begin{array}{l}\text { Search engines, catalogues, } \\
\text { recommendation systems }\end{array}$ & $\begin{array}{c}\text { Virtual, classroom, audio - conferencing, } \\
\text { webcast, white-boarding, video - } \\
\text { conferencing, }\end{array}$ \\
\hline Assistance & Off-line & On-line/Off-line \\
\hline Cooperation Mode & Off-line & Real time \\
\hline Access mode & Unregistered & Registered \\
\hline Costs & Pricing for products & Pricing for courses \\
\hline Support & Informative & Informative, technical, educational \\
\hline Virtual Communities & Informal & formal \\
\hline $\begin{array}{c}\text { Interaction - exchange of } \\
\text { information } \\
\end{array}$ & System - e-consumer & $\begin{array}{c}\text { Instructor - e-learner, system - e-learner, } \\
\text { e-learner - e-learner }\end{array}$ \\
\hline Personalization & $\begin{array}{l}\text { Adapt the content of the e- } \\
\text { commerce system to e- } \\
\text { consumers preferences and } \\
\text { needs }\end{array}$ & $\begin{array}{c}\text { Prescriptive learning where courses } \\
\text { matches to e-learners skills and } \\
\text { knowledge }\end{array}$ \\
\hline
\end{tabular}

\section{Conclusions}

In this work, we reviewed the state of the art in discovering user behavior in both hypermedia contexts and briefly discussed differences and similarities. We argued 
that data mining techniques used in e-commerce should be used as a basis for elearning. In fact, they should be combined with pedagogical approaches and theories.

Although the field of hypermedia adaptation is huge we believe that we made a small contribution to the Endeavour of real adaptation in hypermedia. Our main argument, although simple but overlooked, is that adaptation should be contextspecific and thus be in accordance with the underlying theory. Future work includes a more thorough investigation of social, cultural and economic factors that impose diversity in adaptive hypermedia systems and some recommendations particularly for e-learning and e-commerce applications.

Acknowledgements. This research described in this paper was partly funded by the National Strategic Reference Framework programme 2007-2013, project MIS 296121 "Hellenic Open University".

\section{References}

[1] Stefani, A., Vassiliadis, B., Xenos, M.: Behavioral Patterns in Hypermedia Systems: A Short Study of E-commerce vs. E-learning Practices. In: Sirmakessis, S. (ed.) Adaptive and Personalized Semantic Web. SCI, vol. 14, pp. 57-64. Springer, Heidelberg (2006)

[2] del Ruiz, M., Díaz, M.J., Soler, F.O., Pérez, J.R.: Adaptation in current e-learning systems. Comput. Stand. Interfaces 30(1-2), 62-70 (2008)

[3] Keskustalo, H., Järvelin, K., Pirkola, A.: Evaluating the effectiveness of relevance feedback based on a user simulation model: effects of a user scenario on cumulated gain value. Inf. Retr. 11(3), 209-228 (2008)

[4] Borodin, A., Roberts, G.O., Rosenthal, J.S., Tsaparas, P.: Link analysis ranking: algorithms, theory, and experiments. ACM Transactions on Internet Technology (TOIT) 5(1), 231-297 (2005)

[5] Park, S., Suresh, N.C., Jeong, B.: Sequence-based clustering for Web usage mining: A new experimental framework and ANN-enhanced K-means algorithm. Data Knowl. Eng. 65(3), 512-543 (2008)

[6] Akbulut, Y., Cardak, C.: Adaptive educational hypermedia accommodating learning styles: A content analysis of publications from 2000 to 2011. Comput. Educ. 58(2), 835-842 (2012)

[7] Klašnja-Milićević, A., Vesin, B., Ivanović, M., Budimac, Z.: E-Learning personalization based on hybrid recommendation strategy and learning style identification. Comput. Educ. 56(3), 885-899 (2011)

[8] Magnisalis, I., Demetriadis, S., Karakostas, A.: Adaptive and Intelligent Systems for Collaborative Learning Support: A Review of the Field. IEEE Trans. Learn. Technol. 4(1), 5-20 (2011)

[9] Desmarais, M.C., Baker, R.S.: A review of recent advances in learner and skill modeling in intelligent learning environments. User Modeling and User-Adapted Interaction 22(1-2), 9-38 (2012) 